\title{
Equivalence Classes of Invariant Subspaces in Nonselfadjoint Crossed Products
}

\author{
By \\ Michael McAseY*, Paul S. MuHLY** and Kichi-Suke SAITO****
}

\section{§1. Introdaction}

The invariant subspace structure of nonselfadjoint crossed products was studied in $[2,3,4,5,6,7,8,9,11,13]$. These algebras are certain subalgebras of von Neumann algebras constructed as crossed products of von Neumann algebras by $*$-automorphisms.

In this paper, our setting is the following. Let $M$ be a finite von Neumann algebra with a faithful normal finite trace $\phi$, and let $\alpha$ be a $*$-automorphism of $M$ such that $\phi \circ \alpha=\phi$. Let $L^{2}(M, \phi)$ be the noncommutative $L^{2}$-space associated with $M$ and $\phi$. Then $\alpha$ uniquely extends to a unitary operator $u$ on $\mathbb{L}^{2}(M, \phi)$ such that $\alpha(x)=u x, x \in M$. Form the Hilbert space $\mathbb{L}^{2}=l^{2}(\mathbb{Z}) \otimes L^{2}(M, \phi)$ and consider the operators $L_{x}, x \in M$, and $L_{\delta}$ defined on $\mathbb{L}^{2}$ by the formulae $\mathbb{L}_{x}=I \otimes x$ and $L_{\delta}=S \otimes u$ where $S$ is the usual shift on $l^{2}(\mathbb{Z})$. Then the von Neumann algebra crossed product determined by $M$ and $\alpha$ is defined to be the von Neumann algebra $\mathfrak{L}$ on $\mathbb{L}^{2}$ generated by $\left\{L_{x}: x \in M\right\}(=\mathbb{L}(M))$ and $L_{\delta}$, while the subalgebra which we call a nonselfadjoint crossed product is the $\sigma$-weakly closed subalgebra $\mathfrak{L}_{+}$generated by $L(M)$ and the positive powers of $L_{\delta}$. Let $\mathbb{H}^{2}$ be the subspace $l^{2}\left(\mathbb{Z}_{+}\right) \otimes L^{2}(M, \phi)$ of $\mathbb{L}^{2}$ and let $3(M)$ be the center of $M$. We shall denote by $\operatorname{Lat}\left(\mathfrak{R}_{+}\right)$the lattice of subspaces under $\mathfrak{R}_{+}$such that $\bigcap_{n \geq 0} L_{\delta}^{n} \mathfrak{M}=\{0\}$. If every subspace $\mathfrak{M}$ in $\operatorname{Lat}\left(\mathfrak{S}_{+}\right)$is of the form $\mathfrak{M}=V \mathbb{H}^{2}$, where

Communicated by H. Araki, April 22, 1983. Revised April 2, 1984.

* Department of Mathematics, Bradley University, Peoria, Illinois 61625, U.S.A.

** Department of Mathematics, The University of Iowa, Iowa City, Iowa 52242, U.S.A.

*:** Department of Mathematics, Faculty of Science, Niigata University, Niigata 950-21, Japan.

** The second author was supported in part by a grant from the National Science Foundations.

*** The third author was supported in part by a Grant-in-Aid for Scientific Research from the Japanese Ministry of Education. 
$V$ is a partial isometry in the commutant $\Re$ of $\mathfrak{R}$, we shall say that the BeurlingLax-Halmos theorem (hereafter abbreviated as the BLH theorem) is valid. In [8], we proved that the BLH theorem is valid if and only if $\alpha$ fixes the center $3(M)$ of $M$ elementwise. Therefore, if $\alpha$ does not fix the center $3(M)$ elementwise, then the invariant subspace structure is very complicated. Our aim in this paper is to study it in case $\alpha$ ergodic on $3(M)$.

As the generalization of [7] and [8], Kawamura in [2] has recently studied the invariant subspace structure of a family of shift operators on a Hilbert space, in particular, a family of shift operators given by a nonselfadjoint crossed product (cf. $[3,4]$ ). His setting is not always in standard form. If it is not in standard form, then it is very difficult and very complicated to describe the form of all invariant subspaces. But, if a von Neumann algebra is in standard form, then the underlying Hilbert space has many good informations from the structure of the von Neumann algebra. Therefore, we could investigate the invariant subspace structure of nonselfadjoint crossed products more precisely and so, in this paper we suppose that all von Neumann algebras are in standard form.

In [6], the first author introduced the notion of canonical models for $\operatorname{Lat}\left(\mathfrak{Q}_{+}\right)$. That is, a family of left-full (see $\S 2$ ), invariant subspaces $\left\{\mathfrak{M}_{i}\right\}_{i \in I}$ in $\operatorname{Lat}\left(\mathfrak{R}_{+}\right)$constitutes a complete set of canonical models for $\operatorname{Lat}\left(\mathfrak{Q}_{+}\right)$in case (a) for no two distinct indices $i$ and $j, P_{\mathfrak{M}_{i}}$ is unitary equivalent to $P_{\mathfrak{M}_{j}}$ by a unitary operator in $\mathfrak{R}$; and (b) for every $\mathfrak{M}$ in $\operatorname{Lat}\left(\mathfrak{Q}_{+}\right)$, there is an $i$ in $\mathbb{I}$ and a partial isometry $V$ in $\Re$ such that $V P_{\mathfrak{M}_{i}} V^{*}=\mathbb{P}_{\mathfrak{M} \mathfrak{R}}$, so that $\mathfrak{M}=V \mathfrak{M}_{i}$. The motivation for this concepts stems from the BLH theorem. If $\alpha$ fixes the center $3(M)$ of $M$ elementwise, then the singletone $\left\{\mathbb{H}^{2}\right\}$ is a complete set of canonical models for $\operatorname{Lat}\left(\mathfrak{Q}_{+}\right)$. Let $M=l^{\infty}(X)$, where $X$ is a finite set with elements $t_{0}, t_{1}, \cdots, t_{K-1}$ and let $\alpha$ be a $*$-automorphism of $l^{\infty}(X)$ induced by the permutation $\tau$ of $X$ which is defined by $\tau\left(t_{i}\right)=t_{i+1}(i \neq K-1)$ and $\tau\left(t_{K-1}\right)=t_{0}$. Then, the first author in [6] studied a complete set of canonical models for $\operatorname{Lat}\left(\mathbb{Q}_{+}\right)$which consists of two-sided invariant subspaces of $\mathbb{Z}^{2}$. On the other hand, Solel in [13] studied a complete set of canonical models for $\operatorname{Lat}\left(\mathfrak{Q}_{+}\right)$in case $M=L^{\infty}(X, \mu)$ is nonatomic and $\alpha$ is ergodic on $M$. However, in this case, he also showed that a complete set of canonical models for Lat $\left(\mathfrak{Q}_{+}\right)$does not consist of two-sided invariant subspaces of $\mathbb{L}^{2}$.

In this paper, we consider a complete set of canonical models for $\operatorname{Lat}\left(\mathfrak{R}_{+}\right)$ in the following setting. Let $X$ be a standard Borel space with a finite positive measure $\mu$ and let $\tau$ be an ergodic, invertible measure-preserving transformation on $X$. Let $N$ be a finite factor with a faithful normal tracial state $\phi_{0}$ and let $\alpha_{0}$ 
be a $*$-automorphism of $N$. Then we consider $M=L^{\infty}(X, \mu) \otimes N$ and $\alpha=\tilde{\tau} \otimes \alpha_{0}$ where $\tilde{\tau}$ is a $*$-automorphism of $L^{\infty}(X, \mu)$ induced by $\tau$. Using Solel's idea, we prove that if $N$ is a $\Pi_{1}$-factor, then, for every non-negative measurable function $m$ on $X$ such that $\int_{X} m(t) d \mu(t) \leqq \mu(X)$, there exists a left-full, invariant subspace $\mathfrak{M}$ (of $\mathbb{H}^{2}$ ) in $\operatorname{Lat}\left(\mathfrak{Q}_{+}\right)$with multiplicity function $m$ (Theorem 5.5 ). Therefore, in this case, we choose a complete set of canonical models for $\operatorname{Lat}\left(\mathfrak{Q}_{+}\right)$which consists of left-full, invariant subspaces of $\mathbb{H}^{2}$ in $\operatorname{Lat}\left(\mathfrak{Q}_{+}\right)$corresponding to nonnegative measurable functions such that $\int_{X} m(t) d \mu(t)=\mu(X)$. Further, we consider the case that $N$ is a $\mathbb{I}_{n}$-factor $(n<\infty)$ and that $X$ is a finite set.

In $\S 2$, we define the nonselfadjoint crossed products and invariant subspaces. In $\S 3$, we show that certain equivalence classes of invariant subspaces for $\mathbb{R}_{+}$can be identified with the multiplicity functions of them using the reduction theory in von Neumann algebras. In $\S 4$, we consider the constant fields of finite factors as a preparation of $\S \S 5,6$ and 7 . In $\S 5$, we seek a complete set of canonical models in the case that $\mathbb{L}^{\infty}(X, \mu)$ is non-atomic. In $\S 6$, we consider the case that $X$ is a finite set. Finally, we remark a complete set of canonical models.

\section{§2. Prelimimaries}

Let $M$ be a finite von Neumann algebra acting on a separable Hilbert space $H$. That is, there exists a faithful, normal, finite trace $\phi$ on $M$. Let $L^{2}(M, \phi)$ be the noncommutative $L^{2}$-space associated with $M$ and $\phi([12])$. Let $l_{x}$ (resp. $r_{x}$ ) be the left (resp. right) multiplication on $L^{2}(M, \phi)$ : that is, $l_{x} y=x y$ (resp. $r_{x} y=y x$ ). Put $l(M)=\left\{l_{x}: x \in M\right\}$ and $r(M)=\left\{r_{x}: x \in M\right\}$. Also, we fix once and for all a $*$-automorphism $\alpha$ of $M$ which preserves $\phi$; i.e. $\phi \circ \alpha=\phi$. Thus there is a unitary operator $u$ on $L^{2}(M, \phi)$ induced by $\alpha$. To construct a crossed product, we consider the Hilbert space $\mathbb{E}^{2}$ defined by

$$
\left\{f: \mathbb{Z} \rightarrow L^{2}(M, \phi) \mid \sum_{n \in Z}\|f(n)\|_{2}^{2}<\infty\right\}
$$

where $\|\cdot\|_{2}$ is the norm of $L^{2}(M, \phi)$. For $x \in M$, we define operators $\mathbb{L}_{x}, R_{x}$, $L_{\delta}$ and $R_{\delta}$ on $\mathbb{L}^{2}$ by the formulae

$$
\begin{aligned}
& \left(\mathbb{L}_{x} f\right)(n)=l_{x} f(n), \\
& \left(R_{x} f\right)(n)=r_{\omega^{n}(x)} f(n), \\
& \left(L_{\delta} f\right)(n)=u f(n-1)
\end{aligned}
$$


and

$$
\left(R_{\delta} f\right)(n)=f(n-1) \text {. }
$$

Put $L(M)=\left\{\mathbb{L}_{x}: x \in M\right\}$ and $R(M)=\left\{R_{x}: x \in M\right\}$. We set $\mathscr{L}=\left\{L(M), \mathbb{L}_{\delta}\right\}^{\prime \prime}$ and $\Re=\left\{R(M), R_{\delta}\right\}^{\prime \prime}$ and define the left (resp. right) nonselfadjoint crossed product $\mathfrak{L}_{+}$(resp. $\mathfrak{R}_{+}$) to be the $\sigma$-weakly closed subalgebra of $\mathfrak{R}$ (resp. $\mathfrak{R}$ ) generated by $L(M)$ (resp. $R(M)$ ) and $L_{\delta}$ (resp. $\mathbb{R}_{\delta}$ ).

The automorphism group $\left\{\beta_{t}\right\}_{t \in \mathbb{R}}$ of $\mathfrak{Q}$ dual to $\alpha$ is implemented by the unitary representation of $\mathbb{R},\left\{W_{t}\right\}_{t \in R}$, defined by the formula $\left(W_{t} f\right)(n)=$ $e^{2 \pi i n t} f(n), f \in \mathbb{L}^{2}$; that is, $\beta_{t}(\mathbb{T})=W_{t} T W_{t}^{*}, \mathbb{T} \in \mathbb{\Omega}$, by the definition. It is elementary to check that the spectral resolution of $\left\{W_{t}\right\}_{t \in \mathbb{R}}$ is given by the formula $W_{t}=\sum_{n=-\infty}^{\infty} e^{2 \pi i n t} E_{n}$, where $E_{n}$ is the projection on $\mathbb{L}^{2}$ defined by the formula

$$
\left(E_{n} f\right)(k)= \begin{cases}f(n), & k=n, \\ 0, & k \neq n .\end{cases}
$$

Furthermore, we define $\mathbb{H}^{2}=\left\{j \in \mathbb{L}^{2}: f(n)=0, n<0\right\}$. We refer the reader to [7], [8], [9] and [11] for discussions of these algebras including some of their elementary properties.

Defingition 2.1. Let $\mathfrak{M}$ be a closed subspace of $\mathbb{L}^{2}$. We shall say that $\mathfrak{M}$ is: left-invariant, if $\mathfrak{S}_{+} \mathfrak{M} \subseteq \mathfrak{M}$; left-reducing, if $\mathfrak{M} \subseteq \mathfrak{M}$; left-pure, if $\mathbb{M}$ contains no non-trivial left-reducing subspace; and left-full, if the smallest left-reducing subspace containing $\mathfrak{M}$ is all of $\mathbb{L}^{2}$. The right-hand versions of these concepts are defined similarly, and a closed subspace which is both left and right invariant will be called two-sided invariant.

In this paper, all results will be formulated items of left-invariant subspace. We leave it to the reader to rephrase them to obtain "right-hand" statements.

As in [7] and [8], we are interested in certain wandering subspaces for the bilateral shifts $L_{\delta}$. Let $\mathfrak{M}$ be a left-invariant subspace of $\mathbb{L}^{2}$ and let $P_{\mathfrak{F}}$ be a projection of $\mathbb{L}^{2}$ onto $\mathfrak{F}=\mathfrak{M} \ominus L_{\delta} \mathfrak{M}$. By [7, Proposition 3.1], $P_{\mathfrak{\mho}}$ lies in $\mathbb{L}(M)^{\prime}$. Then we have the following proposition.

Proposition 2.2. Let $\mathfrak{M}$ be a left-invariant subspace of $\mathbb{L}^{2}$ and let $\mathbb{P}_{\widetilde{F}}$ be a projection of $\mathbb{L}^{2}$ onto $\mathfrak{M} \ominus \mathbb{L}_{\delta} \mathfrak{M}$. Then $\mathbb{P}_{\mathscr{F}}$ is a finite projection in $\mathbb{L}(M)^{\prime}$.

Proof. Assume that $P_{\widetilde{F}} \sim P_{1} \leqq P_{\widetilde{F}}$ in $L(M)^{\prime}$. Then there exists a partial isometry $w$ in $L(M)^{\prime}$ such that $w w^{*}=P_{1} \leqq \mathbb{P}_{\Im}$ and $w^{*} w=P_{\Im}$. Put $V=\sum_{n \in Z} L_{\delta}^{n} w L_{\delta}^{*^{n}}$, 
$Q=\sum_{n \in Z} \mathbb{L}_{\delta}^{n} P_{\widetilde{\mho}} \mathbb{L}_{\delta}^{*^{n}}$ and $Q_{1}=\sum_{n \in Z} \mathbb{L}_{\delta}^{n} P_{1} \mathcal{L}_{\delta}^{*^{n}}$, respectively. Then it is clear that $V$ is a partial isometry in $\Re$, and, $Q$ and $Q_{1}$ are projections of $\Re$. Then we have

$$
\begin{aligned}
V Q V^{*} & =\left(\sum_{n \in Z} \mathbb{L}_{\delta}^{n} w L_{\delta}^{*^{n}}\right)\left(\sum_{n \in Z} \mathbb{L}_{\delta}^{n} P_{\overparen{F}} \mathbb{L}_{\delta}^{*^{n}}\right)\left(\sum_{n \in Z} \mathbb{L}_{\delta}^{n} w^{*} L_{\delta}^{*^{n}}\right) \\
& =\sum_{k, m, n \in Z} \mathbb{L}_{\delta}^{n} w \mathbb{L}_{\delta}^{*^{n}} \mathbb{L}_{\delta}^{k} \mathbb{P}_{\widetilde{\mho}} \mathbb{L}_{\delta}^{*^{k}} \mathbb{L}_{\delta}^{m} w^{*} \mathbb{L}_{\delta}^{* m} \\
& =\sum_{k \in Z} \mathbb{L}_{\delta}^{k} w \mathbb{P}_{\overparen{F}} w^{*} \mathbb{L}_{\delta}^{*^{k}}=\sum_{k \in Z} \mathbb{L}_{\delta}^{k} \mathbb{P}_{1} \mathbb{L}_{\delta}^{*^{k}} \\
& =Q_{1} \leqq Q
\end{aligned}
$$

Therefore, $Q \sim Q_{1} \leqq Q$. Since $\Re$ is finite, $Q=Q_{1}$. Thus $P_{\overparen{F}}=P_{1}$. This implies that $\mathbb{P}_{\mathcal{F}}$ is a finite projection in $L\left(M^{\prime}\right)^{\prime}$. This completes the proof.

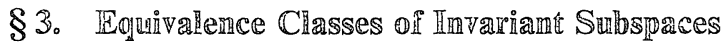

In this section, our aim is to show that the equivalence classes of leftinvariant subspaces of $\mathbb{Z}^{2}$ can be identified with certain equivalence classes of projections as a generalization of [5]. Nioreover, each such a equivalence class can be identified in terms of a multiplicity function. To do this, we use the reduction theory of von Neumann algebras (cf. [1, Part II]).

Let $M$ be a finite von Neumann algebra acting on a separable Hilbert space $H$ and let $3(M)$ be the center of $M$. By [15, III, Proposition 1.21], $3(M)$ is generated by a single selfadjoint operator $h$. We consider the abelian $\mathbb{C}^{*}$ subalgebra $C$ of $B(M)$ which is generated by $\left\{\alpha^{n}(h)\right\}_{n \in Z}$ and $\mathbb{I}_{M}$. Then it is clear that $\mathbb{C}$ is separable and $\alpha(\mathbb{C})=C$. Let $X$ be a maximal ideal space of $C$ such that $C=C(X)$. Then $X$ is a compact metrizable space and there exists a homeomorphism $\tau$ of $X$ onto $X$ such that $\alpha(f)(t)=f\left(\tau^{-1}(\hat{\imath})\right), f \in \mathbb{C}(X)$. Such $\phi$ is a faithful positive linear functional on $C$, there exists a standard finite positive measure $\mu$ on $X$ with support $X$ such that $\phi(f)=\int_{X} f(i) d \mu(t), f \in C$.

Now we consider a direct integral of $M$ with respect to $C$ according to [1]. By [1, Part II, Chapter 6, Theorems 1 and 2], there exists a $\mu$-measurable field $t \rightarrow H(t)$ of nonzero complex Hilbert spaces over $X$, a $\mu$-measurable field $t \rightarrow M(t)$ of factors in the $H(t)^{\prime}$ s and an isomorphism of $\mathbb{H}^{r}$ onto $\int^{\oplus} H(t) d \mu(t)$ which transforms $M$ into $\int^{\oplus} M(t) d \mu(t)$. Therefore, we identify $M, M$ and $B(M)$ with $\int^{\oplus} H(t) d \mu(t), \int^{\oplus} M(t) d \mu(t)$ and the space of diagonal operators, respectively. By [1, Part II, Chapter 5, Corollary of Theorem 2], there exists a $\mu$-measurable field $t \rightarrow \phi_{t}$ of faithful, normal finite traces on $M(t)_{+}$'s such that $\phi=\int \phi_{t} d \mu(t)$. 
Let $L^{2}\left(M, \phi_{t}\right)$ be the noncommutative $L^{2}$-space associated with $M(t)$ and $\phi_{t}$. Then the field $t \rightarrow L^{2}\left(M(t), \phi_{t}\right)$ of complex Hilbert spaces over $X$ is $\mu$-measurable and $L^{2}(M, \phi)=\int L^{2}\left(M(t), \phi_{t}\right) d \mu(t)$. Further, by [1, Part II, Chapter 4, Definition 1], the field $t \rightarrow M(t)$ of archived Hilbert algebras over $X$ in $\int^{\oplus} L^{2}\left(M(t), \phi_{t}\right) d \mu(t)$ is $\mu$-measurable. Let $l_{x(t)}$ (resp. $\left.r_{x(t)}\right)$ be the left (resp. right) multiplication on $L^{2}\left(M(t), \phi_{t}\right)$ and put $l(M(t))=\left\{l_{x(t)}: x(t) \in M(t)\right\}$ (resp. $\left.r(M(t))=\left\{r_{x(t)}: x(t) \in M(t)\right\}\right)$. Then the field $t \rightarrow l(M(t))$ (resp. $t \rightarrow r(M(t))$ ) of factors over $X$ is $\mu$-measurable and $l(M)=\int{ }^{\oplus} l(M(t)) d \mu(t)$ (resp. $r(M)=$ $\left.\int^{\oplus} r(M(t)) d \mu(t)\right)$

Next we define the Hilbert space $\mathbb{L}_{t}^{2}$ by

$$
\mathbb{L}_{t}^{2}=\left\{f_{t}: \mathbb{Z} \rightarrow \mathbb{L}^{2}\left(M(t), \phi_{t}\right) \mid \sum_{n \in \mathbb{Z}}\left\|f_{t}(n)\right\|^{2}<\infty\right\}
$$

and define the operator $L_{x(t)}$ on $\mathbb{L}_{t}^{2}$ by $\left(L_{x(t)} f_{t}\right)(n)=l_{x(t)} f_{t}(n)$. Then the field $t \rightarrow \mathbb{L}_{t}^{2}$ of complex Hilbert spaces over $X$ is $\mu$-measurable and $\mathbb{H}^{2}=\int \mathbb{L}_{t}^{2} d \mu(t)$ and the field $t \rightarrow L(M(t))$ of factors over $X$ is $\mu$-measurable and $L(M)=$ $\int^{\oplus} L(M(t)) d \mu(t)$. Therefore, by [1, Part II, Chapter 3, Theorem 4], the field $t \rightarrow L(M(t))^{\prime}$ of semi-finite factors over $X$ is $\mu$-measurable and $L(M)^{\prime}=$ $\int^{\oplus} L(M(t))^{\prime} d \mu(t)$.

By the definition of $L_{x}\left(\operatorname{resp} . L_{x(t)}\right)$, we may identify $L(M)(\operatorname{resp} . L(M(t)))$ with the von Neumann algebra tensor product $\mathbb{C}_{l^{2}(Z)} \otimes l(M)$ (resp. $\mathbb{C}_{l^{2}(Z)} \otimes$ $l(M(t)))$ where $\mathbb{C}_{l^{2}(\boldsymbol{Z})}$ denotes the algebras of scalar multiples of the identity acting on $l^{2}(\mathbb{Z})$. From this, we can identify the commutant of $L(M)$ :

$$
\begin{aligned}
& L(M)^{\prime}=\left(\mathbb{C}_{l^{2}(Z)} \otimes l(M)\right)^{\prime}=\left(C_{l^{2}(Z)}\right)^{\prime} \otimes l(M)^{\prime} \\
& =B\left(l^{2}(\mathbb{Z})\right) \otimes r(M),
\end{aligned}
$$

where $B\left(l^{2}(\mathbb{Z})\right)$ is the full algebra of operators on $l^{2}(\mathbb{Z})$. Analogously, we can identify the commutant of $L(M(t))$ :

$$
L(M(t))^{\prime}=B\left(l^{2}(\mathbb{Z})\right) \otimes r(M(t)) .
$$

Then, we have $L(M)^{\prime}=\int^{\oplus} B\left(l^{2}(\mathbb{Z})\right) \otimes r(M(t)) d \mu(t)$. Put $\tilde{\phi}\left(r_{x}\right)=\phi(x)$ (resp. $\tilde{\phi}_{t}\left(r_{x(t)}\right)=\phi_{t}(x(t))$. Then $\tilde{\phi}$ (resp. $\left.\tilde{\phi}_{t}\right)$ is a faithful normal finite trace on $r(M)$ (resp. $r(M(t)))$. Let $\operatorname{Tr} \otimes \tilde{\phi}$ (resp. $\operatorname{Tr} \otimes \tilde{\phi}_{t}$ ) be the tensor product of $\operatorname{Tr}$ and $\tilde{\phi}\left(\right.$ resp. $\left.\tilde{\phi}_{t}\right)$ on $B\left(l^{2}(\mathbb{Z})\right) \otimes r(M)\left(\operatorname{resp} . B\left(l^{2}(\mathbb{Z})\right) \otimes r(M(t))\right)$, where $\operatorname{Tr}$ is the canonical trace on $B\left(l^{2}(\mathbb{Z})\right)$. Then $t \rightarrow \operatorname{Tr} \otimes \tilde{\phi}_{t}$ is a $\mu$-measurable field of faithful 
normal semi-finite traces over $X$ and $\operatorname{Tr} \otimes \tilde{\phi}=\int^{\oplus} \operatorname{Tr} \otimes \tilde{\phi}_{t} d \mu(t)$.

Next we define a multiplicity function of a left-invariant subspace. Let $\mathfrak{M}$ be a left-invariant subspace with the wandering subspace $\mathfrak{F}=\mathfrak{M} \ominus \mathbb{L}_{\delta} \mathfrak{M}$. By [7, Proposition 3.1], we know that the projection $\mathbb{P}_{\mathfrak{F}}$ onto the space $\mathfrak{F}$ lies in $L(M)^{\prime}$. By the preceding discussions, we may write $\mathbb{P}=\int^{\oplus} \mathbb{P}(t) d \mu(t)$, where $P(t)$ is a projection in $B\left(l^{2}(\mathbb{Z})\right) \otimes r(M(t))$ for almost all $t$. The multiplicity function of $\mathfrak{M}$ is the function $m$ defined by the equation $m(t)=\left(\operatorname{Tr} \otimes \tilde{\phi}_{t}\right)(P(t))$. Since the field $t \rightarrow P(t)$ of operators is $\mu$-measurable, $m$ is a non-negative measurable function over $X$. Since $P_{\widetilde{F}}$ is finite in $\mathbb{L}(M)^{\prime}$ by Proposition 2.3, $m(t)<\infty$ a.e. Then we have the following theorem as in [5, Theorem 3.4].

Theorem 3.1. For $i=1,2$, let $\mathfrak{M R}_{i}$ be a left-pure, left-invariant subspace of $\mathbb{L}^{2}$ with a multiplicity function $m_{i}$. Let $\mathbb{P}_{\mathfrak{\mho}_{i}}$ be the projection of $\mathbb{L}^{2}$ onto $\mathfrak{\mho}_{i}=$ $\mathfrak{M}_{i} \ominus \mathbb{L}_{\delta} \mathfrak{M}_{i}$. Then the following assertions are equivalent:

(1) there exists a partial isometry $V$ in $\Re$ such that $P_{\mathfrak{M}_{1}}=V P_{\mathfrak{M}_{2}} V^{*}$ where $P_{\mathfrak{M}_{i}}$ is the projection of $\mathbb{L}^{2}$ onto $\mathfrak{M R}_{i}$;

(2) $m_{1}(t) \leqq m_{2}(t)$, a.e.; and

(3) $\boldsymbol{P}_{\widetilde{F}_{1}} \lesssim \mathbb{P}_{\widetilde{F}_{2}}$ in $\mathbb{L}(M)^{\prime}$.

Furthermore, if the condition (1) is satisfied, then $\mathfrak{M}_{1}=V \mathfrak{M C}_{2}$.

Proof. (1) $\rightarrow(2)$. Since $\mathfrak{R} \subset \mathbb{L}(M)^{\prime}, V$ is a decomposal operator. Thus $V=\int^{\oplus} V(t) d \mu(t)$, where $V(t)$ is a partial isometry in $B\left(l^{2}(\mathbb{Z})\right) \otimes r(M(t))$ for almost everywhere $t$. Since $P_{\mathfrak{F}_{i}} \in \mathbb{L}(M)^{\prime}, P_{\mathfrak{F}_{i}}=\int^{\oplus} P_{i}(t) d \mu(t)$, where $\mathbb{P}_{i}(t)$ is a projection of $B\left(l^{2}(\mathbb{Z})\right) \otimes r(M(t))$ for almost all $t$. By (1), it is clear that $P_{\widetilde{F}_{1}}=V P_{\widetilde{F}_{2}} V^{*}$. Therefore, $P_{1}(t)=V(t) P_{2}(i) V(t)^{*}$, a.e. and so $m_{1}(t)=\left(\operatorname{Tr} \otimes \tilde{\phi}_{t}\right)$ 。 $\left(P_{1}(t)\right)=\left(\operatorname{Tr} \otimes \tilde{\phi}_{t}\right)\left(V(t) P_{2}(t) V(t)^{*}\right) \leqq\left(\operatorname{Tr} \otimes \tilde{\phi}_{t}\right)\left(P_{2}(t)\right)=m_{2}(t)$. Thus $m_{1}(t) \leqq m_{2}(t)$ a.e.

$(2) \rightarrow(3)$. Since $m_{1}(t) \leqq m_{2}(t)$, a.e., by [1, Part $\mathbb{I I}$, Chapter 2, Proposition 13], $\mathbb{P}_{1}(t) \lessgtr \mathbb{P}_{2}(t)$, a.e. Therefore, by [1, Part III, Chapter 1, Exercise 15], $\mathbb{P}_{\mho_{1}} \lessgtr \mathbb{P}_{\widetilde{F}_{2}}$ in $L(M)^{\prime}$ 。

(3) $\rightarrow(1)$. It is clear by [7, Theorem 3.2]. This completes the proof.

Further, as in [13, Proposition 2.3], we can easily prove the following proposition and so the proof is omitted.

Proposition 3.2. Keep the notations and assumptions as in Theorem 3.1. Then the following assertions are equivalent: 
(1) there exists a partial isometry $V$ in $\Re$ such that $P_{\mathfrak{M}_{1}}=V P_{\mathfrak{M}_{2}} V^{*}$ and $V^{*} V=\sum_{n \in \mathbb{Z}} \mathbb{L}_{\delta}^{n} P_{\mho_{2}} \mathbb{L}_{\delta}^{*^{n}}$

(2) $m_{1}(t)=m_{2}(t)$ a.e.; and

(3) $P_{\mathfrak{\mho}_{1}} \sim P_{\mathfrak{F}_{2}}$ in $\mathbb{L}(M)^{\prime}$.

\section{\$4. Constant Fields of Finite Fretors}

In this section, we consider the constant fields of finite factors over $X$, that is, the tensor product of $L^{\infty}(X, \mu)$ and a finite factor $N$.

Let $X$ be a compact metrizable space with a finite measure $\mu$. Let $\tau$ be an invertible, ergodic, measure-preserving transformation on $X$. Let $N$ be a finite factor with a faithful, normal tracial state $\phi_{0}$, and let $\alpha_{0}$ be a $*$-automorphism of $N$. Put $\tilde{\tau}(f)(t)=f\left(\tau^{-1} t\right), t \in X$. Let $\tilde{\tau} \otimes \alpha_{0}$ be the tensor product of $\tilde{\tau}$ and $\alpha_{0}$ on $L^{\circ}(X, \mu) \otimes N$. Put $M=L^{\infty}(X, \mu) \otimes N, \alpha=\tilde{\tau} \otimes \alpha_{0}$ and $\phi=\int_{X} \cdot d \mu(t) \otimes \phi_{0}$, respectively. Let $L^{2}\left(N, \phi_{0}\right)$ be the noncommutative $L^{2}$-space associated with $N$ and $\phi_{0}$. We denote the operators in the left regular representation of $N$ onto $L^{2}\left(N, \phi_{0}\right)$ by $l_{x}, x \in N$, and those in the right regular representation by $r_{x}$. Put $l(N)=\left\{l_{x}: x \in N\right\}$ and $r(N)=\left\{r_{x}: x \in N\right\}$. Since $\phi_{0} \circ \alpha_{0}=\phi_{0}$, there is uniquely a unitary operator $u_{0}$ on $L^{2}\left(N, \phi_{0}\right)$ induced by $\alpha_{0}$. In this paper, we identify $L^{\infty}(X, \mu) \otimes N$ with $L^{\infty}(X, N)$. Using the product of the counting measure on $\mathbb{Z}$ and the measure $\mu$ on $X$, we can realize $\mathbb{Z} \times X$ as a measure space. Then we can identify $\mathbb{L}^{2}$ with

$$
\left\{f: \mathbb{Z} \times X \rightarrow L^{2}\left(N, \phi_{0}\right) \mid \sum_{n \in Z} \int_{X}\|f(n, \hat{\imath})\|^{2} d \mu(t)<\infty\right\},
$$

where $\|\cdot\|$ is the norm of $L^{2}\left(N, \phi_{0}\right)$. For $x \in M$, we can calculate $L_{x}, R_{x}, L_{\delta}$ and $R_{\delta}$ on $\mathbb{L}^{2}$ as follows;

$$
\begin{aligned}
& \left(\mathbb{L}_{x} f\right)(n, t)=l_{x(t)} f(n, t), \\
& \left(R_{x} f\right)(n, t)=r_{\alpha_{0}{ }^{n}\left(x\left(\tau^{-n} t\right)\right)} f(n, t), \\
& \left(L_{\delta} f\right)(n, t)=u_{0} f\left(n-1, \tau^{-1} t\right)
\end{aligned}
$$

and

$$
\left(R_{\delta} f\right)(n, t)=f(n-1, t), \quad f \in \mathbb{L}^{2} .
$$

We recall that the space $\mathbb{L}^{2}$ may be identified with the direct integral $\int^{\oplus} l^{2}(\mathbb{Z}) \otimes L^{2}\left(N, \phi_{0}\right) d \mu(t)$. Since $L^{\infty}(X, \mu)$ is a maximal abelian von Neumann algebra on $\mathbb{L}^{2}(X, \mu)$, we can identify the commutant of $L(M)$; that is, 


$$
\begin{aligned}
L(M)^{\prime} & =\left(L^{\infty}(X, \mu) \otimes \mathbb{C}_{l^{2}(Z)} \otimes l(N)\right)^{\prime} \\
& =L^{\infty}(X, \mu) \otimes B\left(l^{2}(\mathbb{Z})\right) \otimes r(N) \\
& =\int^{\oplus}\left(B\left(l^{2}(\mathbb{Z})\right) \otimes r(N)\right) d \mu(t) .
\end{aligned}
$$

Put $\tilde{\phi}_{0}\left(r_{x}\right)=\phi_{0}(x), x \in N$. We consider the tensor product $\operatorname{Tr} \otimes \tilde{\phi}_{0}$ of $\operatorname{Tr}$ and $\tilde{\phi}_{0}$. Then $\operatorname{Tr} \otimes \tilde{\phi}_{0}$ is a faithful normal semifinite trace on $B\left(l^{2}(\mathscr{Z})\right) \otimes r(N)$. Thus, as in $\S 3$, we can define the multiplicity function of invariant subspaces.

\section{§5. Case: $\mathbb{L}^{\infty}(x, \mu)$ is Nom-Atomic}

Keep the notations in $\S 4$. Throughout this section, we suppose that $L^{\infty}(X, \mu)$ is non-atomic. Let $\chi_{E}$ be a characteristic function of a measurable subset $E$ in $X$ and let $p$ be a projection of $N$. We define a projection $p$ in $L(M)^{\prime}$ by

$$
(P f)(n, t)= \begin{cases}\chi_{E}(t) r_{p} f(0, t), & n=0, \\ 0, & n \neq 0 .\end{cases}
$$

Since $P \leqq E_{0}$, where $E_{0}$ is defined in $\S 2$, and since $\left\{L_{\delta}^{n} E_{0} L_{\delta}^{*^{n}}\right\}_{n \in Z}$ is mutually orthogonal, $\left\{L_{\delta}^{n} P L_{\delta}^{*^{n}}\right\}_{n \in Z}$ is mutually orthogonal. Thus, we define a closed subspace $\mathfrak{M}(E, p)$ of $\mathbb{L}^{2}$ by $\mathfrak{M}(E, p)=\sum_{n=0}^{\infty}\left(L_{\delta}^{n} P L_{\delta}^{*^{n}}\right) \mathbb{L}^{2}$. Then we present in

Lemma 5.1. (1) $\mathfrak{M}(E, p)$ is a left-pure, left-invariant subspace of $\mathbb{H}^{2}$ with the multiplicity function $\chi_{E}(t) \phi_{0}(p)$.

(2) $\operatorname{Me}(E, p)=\left[\mathfrak{Q}_{+}(P \psi)\right]_{2}$, where $\left[{ }^{\circ}\right]_{2}$ is the closed linear span of $\mathfrak{Z}_{+}(P \psi)$ in $\mathbb{L}^{2}$ and $\psi(k, t)=\delta_{k, 0} 1$.

Proof. (1) is clear by the definition of $\mathfrak{M E}(E, p)$.

(2) Since $(P \psi)(0, t)=\chi_{E}(t) p$ and $(P \psi)(n, t)=0, n \neq 0$, we have

$$
\begin{aligned}
& \left(\sum_{n=0}^{\infty} L_{\delta}^{n} P L_{\delta}^{*^{n}} P \psi\right)(k, t)=\sum_{n=0}^{\infty} u_{0}^{n}\left(P L_{\delta}^{*^{n}} P \psi\right)\left(k-n, \tau^{-n} t\right) \\
& =u_{0}^{k} \chi_{E}\left(\tau^{-k} t\right) r_{p}\left(L_{\delta}^{*^{k}} P \psi\right)\left(0, \tau^{-k} t\right) \\
& =\chi_{E}\left(\tau^{-k} t\right) u_{0}^{k} r_{p} u_{0}^{*^{k}}(P \psi)(k, t) \\
& =\chi_{E}\left(\tau^{-k} t\right) r_{\alpha_{0}^{k}(p)^{\delta} \delta_{k, 0}} \chi_{E}(t) p \\
& =\delta_{k, 0} \chi_{E}(t) p=(P \psi)(k, t)
\end{aligned}
$$

Thus $P \psi \in \mathfrak{M}(E, p)$ and so $\left[\mathfrak{R}_{+} P \psi\right]_{2} \subset \mathfrak{M}(E, p)$. Let $f \in \mathfrak{M}(E, p) \cap \mathbb{L}^{\infty}$, where $\mathbb{L}^{\infty}=\Omega \psi$. Put $f_{k}(\hat{\imath})=f(k, t) \in M$. Then we have 


$$
\begin{aligned}
& \sum_{k=0}^{\infty}\left(L_{f_{k}} L_{\delta}^{k} P \psi\right)(n, t)=\sum_{k=0}^{\infty} l_{f_{k}(t)} u_{0}^{k}(P \psi)\left(n-k, \tau^{-k} t\right) \\
& =l_{f_{n}(t)} u_{0}^{n} \chi_{E}\left(\tau^{-n} t\right) p=\chi_{E}\left(\tau^{-n} t\right) r_{\alpha_{0}^{n}(p)} f(n, t) \\
& =\left(\sum_{k=0}^{\infty} L_{\delta}^{k} P L_{\delta}^{*^{k}} f\right)(n, t)=f(n, t) .
\end{aligned}
$$

Therefore, $\mathfrak{M}(E, p) \cap L^{\infty} \subset\left[\mathfrak{Q}_{+} P \psi\right]_{2}$. By [10, Theorem 1], we have $\mathfrak{M}(E, p)=$ $\left[\Omega_{+} P \psi_{2}\right.$. This completes the proof.

Since $\tau$ is ergodic on $X$, we have the following lemma.

Lemma 5.2 ([13, Lemma 3.3]). Let $E, F$ be measurable sets of $X$ with $\mu(E)=$ $\mu(F)$. Then there are measurable subsets $\left\{E_{n}\right\}_{n=0}^{\infty}$ and $\left\{F_{n}\right\}_{n=0}^{\infty}$ satisfying:

(1) $E=\sum_{n=0}^{\infty} E_{n}$ and $F=\sum_{n=0}^{\infty} F_{n}$; and

(2) $F_{n}=\tau^{n}\left(E_{n}\right)$ for each $n \geqq 0$.

Lemma 5.3. Keep the notations as in Lemma 5.2. Let $p$ be a projection of N. Then $U=\sum_{k=0}^{\infty} L_{\chi_{F_{k}} \alpha_{0}^{k}(p)} L_{\delta}^{k}$ is a partial isometry in $\mathfrak{L}_{+}$with initial projection $L_{\chi_{E} p}$ and final projection $\sum_{k=0}^{\infty} L_{\chi_{F_{k}}} \alpha_{0}^{k}(\not)^{\circ}$

Proof. Since $L_{\delta}^{n} L_{\chi_{F} p} L_{\delta}^{*^{n}}=L_{\chi_{\tau^{n}(F)} \alpha_{0}^{n}(p)}$ and $L_{\chi_{F_{k}}} \alpha_{0}^{k}(p) L_{\chi_{F j}} \alpha_{0}^{j}(p)=0$ for $k \neq j$, we have by Lemma 5.2 ,

$$
\begin{aligned}
U^{*} U & =\sum_{k=0}^{\infty} \sum_{j=0}^{\infty} L_{\delta}^{*^{k}} L_{\chi_{F_{k}}} \alpha_{0}^{k}(p) L_{\chi_{F j} \alpha_{0}^{j}(p)} L_{\delta}^{j} \\
& =\sum_{k=0}^{\infty} L_{\delta}^{*^{k}} L_{\chi_{F_{k}}} \alpha_{0}^{k}(p) \\
& =\sum_{k=0}^{\infty} L_{\chi_{E_{k}} p}=\sum_{k=0}^{\infty} L_{\chi_{\tau^{-k}\left(F_{k}\right)} p} L_{\chi_{E}} p^{\circ}
\end{aligned}
$$

Since $L_{\delta}^{*^{k}} L_{\chi_{F_{k}}} \alpha_{0}^{k}(p) L_{\delta}^{k} L_{\delta}^{*^{j}} L_{\chi_{F_{j}}} \alpha_{0}^{j}(p)^{2} L_{\delta}^{j}=0$ for $k \neq j$, we analogously have

$$
U U^{*}=\sum_{k=0}^{\infty} \sum_{j=0}^{\infty} L_{\chi_{F_{k}}} \alpha_{0}^{k}(p) L_{\delta}^{k} L_{\delta}^{*^{j}} L_{\chi_{F}} \alpha_{0}^{j}(p)=\sum_{k=0}^{\infty} L_{\chi_{F_{k}}} \alpha_{0}^{k}(p) \cdot
$$

This completes the proof.

Lemma 5.4. Let $E, F$ be measurable subsets of $X$ such that $\mu(E)=\mu(F)$ and let $p$ be a projection of $N$. Then there exists a left-pure, left-invariant subspace $\mathfrak{M}$ of $\mathfrak{M}(E, p)$ such that the multiplicity function of $\mathfrak{M}$ is $\chi_{F}(t) \phi_{0}(p)$ and 
$\sum_{n \in Z} L_{\delta}^{n} P_{\mathfrak{F}} L_{\delta}^{*^{n}}=R_{\chi_{E} p}$ where $\mathfrak{F}=\mathfrak{M C} \ominus L_{\delta} \mathfrak{M}$

Proof. As in Lemma 5.2, we take measurable subsets $\left\{E_{n}\right\}_{n=0}^{\infty}$ and $\left\{F_{n}\right\}_{n=0}^{\infty}$ Hence, by Lemma 5.3, we define a partial isometry $U=\sum_{k=0}^{\infty} L_{\chi_{F_{k}}} \alpha_{0}^{k}(p) L_{\delta}^{k}$ in $\mathfrak{Q}_{+}$. Let $e_{0} \in \mathbb{L}^{2}$ be defined by: $e_{0}(k, t)=\chi_{E}(t) p, k=0$, and $e_{0}(k, t)=0, k \neq 0$. Then we have, for $k \geqq 0$,

$$
\begin{aligned}
\left(U e_{0}\right)(k, t) & =\left(\sum_{n=0}^{\infty} L_{\chi_{F_{n}} \alpha_{0}^{n}(p)} L_{\delta}^{n} e_{0}\right)(k, t) \\
& =\sum_{n=0}^{\infty} \chi_{F_{n}}(t) \alpha_{0}^{n}(p)\left(L_{\delta}^{n} e_{0}\right)(k, t) \\
& =\sum_{n=0}^{\infty} \chi_{F_{n}}(t) \alpha_{0}^{n}(p) u_{0}^{n} e_{0}\left(k-n, \tau^{-n} t\right) \\
& =\chi_{F_{k}}(t) \alpha_{0}^{k}(p) u_{0}^{k} \chi_{E}\left(\tau^{-k} t\right) p \\
& =\chi_{F_{k}}(t) \alpha_{0}^{k}(p) \chi_{\tau^{k}(E)}(t) \alpha_{0}^{k}(p) \\
& =\chi_{F_{k}}(t) \alpha_{0}^{k}(p)
\end{aligned}
$$

Thus we define a projection $P_{1}$ in $L(M)^{\prime}$ by

$$
\left(P_{1} f\right)(k, t)= \begin{cases}\chi_{F_{k}}(t) r_{\alpha_{0}^{k}(p)} f(k, t), & k \geqq 0, \\ 0, & k<0,\end{cases}
$$

for $f \in \mathbb{L}^{2}$. Then it is clear that $\left(L_{\delta}^{m} P_{1} L_{\delta}^{*^{m}}\right)\left(L_{\delta}^{n} P_{1} L_{\delta}^{*^{n}}\right)=0$, for $n, m \in \mathbb{Z}(n \neq m)$. This implies that $P_{1}$ is a wandering projection in $L(M)^{\prime}$. Therefore, we define a closed subspace $\mathfrak{M}$ by $\mathfrak{M}=\left(\sum_{n=0}^{\infty} L_{\delta}^{n} P_{1} L_{\delta}^{*^{n}}\right) \mathbb{L}^{2}$. Then it is clear that $\mathfrak{M}$ is leftpure and left-invariant. Further,

$$
\begin{aligned}
& \left(\operatorname{Tr} \otimes \tilde{\phi}_{0}\right)\left(P_{1}(t)\right)=\sum_{k=0}^{\infty} \chi_{F_{k}}(t) \phi_{0}\left(\alpha_{0}^{k}(p)\right) \\
& =\sum_{k=0}^{\infty} \chi_{F_{k}}(t) \phi_{0}(p)=\chi_{F}(t) \phi_{0}(p) .
\end{aligned}
$$

Thus, the multiplicity function $m$ of $\mathfrak{M}$ is $\chi_{F}(t) \phi_{0}(p)$.

Next we shall show that $\mathfrak{M}=\left[\mathfrak{Q}_{+}\left(U e_{0}\right)\right]_{2}$, where $\left[\mathbb{Q}_{+} U e_{0}\right]_{2}$ is the closed linear span of $\mathfrak{Q}_{+} U e_{0}$ in $\mathbb{Z}^{2}$. At first, we have, for $k \geqq n \geqq 0$,

$$
\begin{aligned}
& \left(L_{\delta}^{n} P_{1} L_{\delta}^{*^{n}}\right)\left(U e_{0}\right)(k, t)=u_{0}^{n}\left(P_{1} L_{\delta}^{*^{n}} U e_{0}\right)\left(k-n, \tau^{-n} t\right) \\
& =u_{0}^{n} \chi_{F_{k-n}}\left(\tau^{-n} t\right) r_{\alpha_{0}^{k-n}(p)}\left(L_{\delta}^{*^{n}} U e_{0}\right)\left(k-n, \tau^{-n} t\right) \\
& =\chi_{F_{k-n}}\left(\tau^{-n} t\right) u_{0}^{n} r_{\alpha_{0}^{k-n}(p)} u_{0}^{*^{n}}\left(U e_{0}\right)(k, t) \\
& =\chi_{F_{k-n}}\left(\tau^{-n} t\right) r_{\alpha_{0}^{k}(p)}\left(U e_{0}\right)(k, t)
\end{aligned}
$$




$$
\begin{aligned}
& =\chi_{\tau^{k}\left(E_{k-n}\right)}(t) r_{\alpha_{0}^{k}(p)} \chi_{F_{k}}(t) \alpha_{0}^{k}(p) \\
& =\chi_{\tau^{k}\left(E_{k-n}\right)}(t) \chi_{\tau^{k}\left(E_{k}\right)}(t) \alpha_{0}^{k}(p) \\
& =\delta_{k-n, k} \chi_{\tau^{k}\left(E_{k}\right)}(t) \alpha_{0}^{k}(p)
\end{aligned}
$$

Since $\left(L_{\delta}^{n} P_{1} L_{\delta}^{*^{n}}\right)\left(U e_{0}\right)(k, t)=0$, for $n>k$, we have, for $k \geqq 0$,

$$
\begin{aligned}
& \left(\sum_{n=0}^{\infty} L_{\delta}^{n} P_{1} L_{\delta}^{*^{n}}\right)\left(U e_{0}\right)(k, t)=\sum_{n=0}^{k} L_{\delta}^{n} P_{1} L_{\delta}^{*^{n}}\left(U e_{0}\right)(k, t) \\
& =\sum_{n=0}^{k} \delta_{k-n, k} \chi_{\tau^{k}\left(E_{k}\right)}(t) \alpha_{0}^{k}(p)=\chi_{\tau^{k}\left(E_{k}\right)}(t) \alpha_{0}^{k}(p) \\
& =\left(U e_{0}\right)(k, t)
\end{aligned}
$$

Thus $U e_{0} \in \mathfrak{M}$ and so $\left[\mathbb{R}_{+} U e_{0}\right]_{2} \subset \mathfrak{M}$. Further, we note that $f \in \mathfrak{M}$ if and only if $\sum_{n=0}^{k} \chi_{\tau^{k}\left(E_{k-n}\right)}(t) r_{\alpha_{0}^{k}(p)} f(k, t)=f(k, t), k \geqq 0$, and $f(k, t)=0, k<0$. By [10, Theorem 1], $\mathfrak{M} \cap \mathbb{L}^{\infty}$ is dense in $\mathfrak{M}$. Thus, if $g \in \mathfrak{M} \cap \mathbb{H}^{\infty}$, then we put

$$
g_{k, n}(t)=\chi_{\tau^{k}\left(E_{k-n}\right)}(t) r_{\alpha_{0}^{k}(p)} g(k, t) \in N
$$

Thus $g_{k, n} \in M$. Then $\sum_{n=0}^{k} g_{k, n}(t)=g(k, t)$. Hence we have for $m, k \geqq n \geqq 0$,

$$
\begin{aligned}
& \left(L_{g_{k, n}} L_{\delta}^{n}\left(U e_{0}\right)\right)(m, t)=g_{k, n}(t) u_{0}^{n}\left(U e_{0}\right)\left(m-n, \tau^{-n} t\right) \\
& =g_{k, n}(t) u_{0}^{n}\left(\chi_{F_{m-n}}\left(\tau^{-n} t\right) \alpha_{0}^{m-n}(p)\right) \\
& =g_{k, n}(t) \chi_{\tau^{n}\left(F_{m-n}\right)}(t) \alpha_{0}^{m}(p) \\
& =\chi_{\tau^{k}\left(E_{k-n}\right)}(t) \chi_{\tau^{n}\left(F_{m-n}\right)}(t) r_{\alpha_{0}^{k}(p)} g(k, t) \alpha_{0}^{m}(p) \\
& =\chi_{\tau^{k}\left(E_{k-n}\right)}(t) \chi_{\tau^{m}\left(E_{m-n}\right)}(t) r_{\alpha_{0}^{k}(p)} g(k, t) \alpha_{0}^{m}(p) \\
& =\delta_{k, m} \chi_{\tau^{m}\left(E_{m-n}\right)} g(m, t) \alpha_{0}^{m}(p)=\delta_{k, m} g_{m, n}(t) .
\end{aligned}
$$

Thus we have

$$
\begin{aligned}
& \left(\sum_{k=0}^{\infty} \sum_{n=0}^{k}\left(\mathbb{L}_{g_{k, n}} \mathbb{L}_{\delta}^{n}\left(U e_{0}\right)\right)\right)(m, t)=\sum_{k=0}^{\infty} \sum_{n=0}^{k} \delta_{k, m} g_{m, n}(t) \\
& =\sum_{k=0}^{\infty} \delta_{k, m} \sum_{n=0}^{k} g_{m, n}(t)=\sum_{n=0}^{m} g_{m, n}(t)=g(m, t) .
\end{aligned}
$$

Hence this implies that $\mathfrak{M} \cap \mathbb{L}^{\infty} \subset\left[\mathfrak{R}_{+}\left(U e_{0}\right)\right]_{2}$ and so $\mathfrak{M} \subset\left[\mathfrak{R}_{+}\left(U e_{0}\right)\right]_{2}$. Therefore, $\mathfrak{M}=\left[\mathfrak{R}_{+}\left(U e_{0}\right)\right]_{2}$.

Since $U \in \mathfrak{R}_{+}$, by Lemma 5.1, $\mathfrak{M} \subset\left[\mathfrak{Q}_{+} e_{0}\right]_{2}=\mathfrak{M}(E, p)$. Further, since $\left(L_{\delta}^{m} P_{1} L_{\delta}^{*^{m}} f\right)(k, t)=0$, for $k>m$, we have 


$$
\begin{aligned}
& \left(\sum_{m=-\infty}^{\infty} L_{\delta}^{m} P_{1} L_{\delta}^{*^{m}} f\right)(k, t) \\
& =\sum_{m=-\infty}^{k} \chi_{\tau^{k}\left(E_{k-m}\right)}(t) r_{\alpha_{0}^{k}(p)} f(k, t) \\
& =\sum_{m=0}^{\infty} \chi_{\tau^{k}\left(E_{m}\right)}(t) r_{\alpha_{0}^{k}(p)} f(k, t) \\
& =\chi_{\tau^{k}(E)}(t) r_{\alpha_{0}^{k}(p)} f(k, t) \\
& =\chi_{E}\left(\tau^{-k}(t)\right) r_{\alpha_{0}^{k}(p)} f(k, t)=\mathbb{R}_{\chi_{E} p} f(k, t) .
\end{aligned}
$$

This completes the proof.

As in [13, Lemma 3.1], we have the following lemma.

Lemma 5.5. Let $\left\{\mathfrak{M l}_{i}\right\}_{i \in I}$ is a finite or countable collection of left-pure, left-invariant subspaces such that $\mathfrak{M R}_{i}$ is orthogonal to $\mathfrak{M E}_{j}$, for $i \neq j$. Let $m_{i}(i)$ be the multiplicity function of $\mathfrak{M R}_{i}$. Then $\mathfrak{M}=\sum_{i \in I} \oplus \mathfrak{M}_{i}$ is a lefi-pure, leftinvariant subspace with multiplicity function $m(t)=\sum_{i \in I} m_{i}(t)$, a.e.

Next we consider two cases, that is, (1) $N$ is a $\mathbb{I}_{1}$-factor and (2) $N$ is a $\mathbb{I}_{n}$ factor, $n<\infty$. Iet $\mathfrak{M}$ be a left-pure, left-invariant subspace of $\mathbb{H}^{2}$ with the multiplicity function $m(t)$. If $N$ is a finite factor, then $m(t) \in \mathbb{R}_{+}=\{s \in \mathbb{R}:$ $s \geqq 0\}$, a.e. In particular, if $N$ is a $\mathbb{I}_{n}$-factor, $n<\infty$, then $m(t) \in(1 / n) \mathbb{Z}_{+}=$ $\left\{k / n: k \in \mathbb{Z}_{+}\right\}$. For, since $N$ is a $\mathbb{I}_{n}$-factor, $N$ is identified with an $n \times n$ matrix algebra $M_{n}$ on a Hilbert space $K(\operatorname{dim} K=n)$. Then $\mathbb{R}\left(l^{2}(\mathbb{Z})\right) \otimes r(N)=$ $B\left(l^{2}(\mathbb{Z})\right) \otimes M_{n}=B\left(l^{2}(\mathbb{Z}) \otimes K\right)$. Since $\operatorname{Tr} \otimes n \tilde{\phi}_{0}$ is the canonical trace on $B\left(l^{2}(\mathbb{Z}) \otimes K\right),\left(\operatorname{Tr} \otimes n \tilde{\phi}_{0}\right)\left(P_{\widetilde{⿰}}(t)\right) \in \mathbb{Z}_{+}$. Since $\operatorname{Tr} \otimes n \tilde{\phi}_{0}=n\left(\operatorname{Tr} \otimes \tilde{\phi}_{0}\right), m(t) \in(1 / n) \mathbb{Z}_{+}$. Conversely, we present in

Theorem 5.6. (1) Let $N$ be a II $I_{1}$-factor and let $m(i)$ be a measurable function on $X$ with values in $\mathbb{R}_{+}$such that $\int_{X} m(t) d \mu(t) \leqq \mu(X)$. Then there exists a left-pure, left-invariant subspace $\mathfrak{N e}$ of $\mathbb{H}^{2}$ with multiplicity function $m(t)$. Moreover, if $\int_{X} m(t) d \mu(t)=\mu(X)$, then $\mathfrak{M}$ is left-full.

(2) Let $N$ be a $\mathbb{I}_{n}$-factor and let $m(t)$ be a measurable function on $X$ with values in $(1 / n) \mathbb{Z}_{+}$such that $\int_{X} m(t) d \mu(t) \leqq \mu(X)$. Then there exists a left-pure, left-invariant subspace $\mathfrak{M}$ of $\mathbb{H H}^{2}$ with multiplicity function $m(t)$. Moreover, if $\int_{X} m(t) d \mu(t)=\mu(X)$, then $\mathfrak{M}$ is left-full.

Proof. (1) Suppose that $N$ is a $\mathbb{I}_{1}$-factor. Let $m$ be a measurable 
function on $X$ with values in $\mathbb{R}_{+}$such that $\int_{X} m(t) d \mu(t) \leqq \mu(X)$. Then there exist measurable subsets $\left\{E_{n}\right\}_{n=1}^{\infty}$ and $\left\{G_{n}\right\}_{n=1}^{\infty}$ of $X$ such that

$$
m(t)=\sum_{n=1}^{\infty} \chi_{E_{n}}(t)+\sum_{n=1}^{\infty}(1 / 2)^{n} \chi_{G_{n}}(t) .
$$

Since $\mu$ is non-atomic, there exist measurable subsets $\left\{F_{n}\right\}_{n=1}^{\infty}$ such that $\mu\left(E_{n}\right)=\mu\left(F_{n}\right)$ and $F_{n} \cap F_{m}=\phi, n \neq m$. As in the proof of [13, Theorem 3.6], by Lemma 5.4, there exists a left-pure left-invariant subspace $\mathfrak{M}_{n}$ of $\mathfrak{M}\left(F_{n}, 1\right)$ such that the multiplicity function of $\mathfrak{M}_{n}$ is $\chi_{E_{n}}(t)$ and $\sum_{n \in Z} L_{\delta}^{n} P_{\mho_{n}} L_{\delta}^{*^{n}}=R_{\chi_{F_{n}}}$, where $\mathfrak{F}_{n}=\mathfrak{M}_{n} \ominus L_{\delta} \mathfrak{M}_{n}$

Next, since $\mu$ is nonatomic, there exist mutually disjoint measurable subsets $\left\{G_{n, i}\right\}_{i=1}^{2^{n}}$ such that $G_{n}=G_{n, 1}+G_{n, 2}+\cdots+G_{n, 2^{n}}$ and $\mu\left(G_{n, j}\right)=\left(1 / 2^{n}\right) \mu\left(G_{n}\right), 1 \leqq$ $j \leqq 2^{n}$. Since $N$ is a $\mathbb{I}_{1}$-factor, there exist mutually orthogonal equivalent projections $\left\{p_{n, j}\right\}_{j=1}^{2^{n}}$ of $N$ such that $\sum_{j=1}^{2^{n}} p_{n, j}=1$. Since $\mu$ is non-atomic, there exist mutually disjoint measurable subsets $\left\{H_{n}\right\}_{n=1}^{\infty}$ such that $\sum_{n=1}^{\infty} H_{n} \subset X-\sum_{n=1}^{\infty} F_{n}$ and $\left(1 / 2^{n}\right) \mu\left(G_{n}\right)=\mu\left(H_{n}\right)$. By Lemma 5.4, there exists a left-pure, left-invariant subspace $\mathfrak{R}_{n, j}$ of $\mathfrak{M}\left(H_{n}, p_{n, j}\right)$ such that the multiplicity function of $\mathfrak{R}_{n, j}$ is $\chi_{G_{n, j}}(t)\left(1 / 2^{n}\right)$ and $\sum_{n \in Z} L_{\delta}^{n} P_{\mathfrak{B}_{n, j}} L_{\delta}^{*^{n}}=R_{\chi_{B_{n}} p_{n, j}}$, where $\mathfrak{B}_{n, j}=\mathfrak{N}_{n, j} \ominus L_{\delta} \mathfrak{\mathfrak { N } _ { n , j }}$. Put $\mathfrak{N}_{n}=\sum_{j=1}^{2^{n}} \oplus \mathfrak{N}_{n, j}$. By Lemma $5.5, \mathfrak{R}_{n}$ is a left-pure, left-invariant subspace of $\sum_{j=1}^{2^{n}} \oplus \mathfrak{M}\left(H_{n}, p_{n, j}\right)\left(=\mathfrak{M}\left(H_{n}, 1\right)\right)$ with multiplicity function $\chi_{G_{n}}(t)\left(1 / 2^{n}\right)$ such that $\sum_{n \in Z} L_{\delta}^{n} P_{\mathfrak{B}_{n}} L_{\delta}^{*^{n}}=R_{\chi_{H_{n}}}$, where $\mathfrak{B}_{n}=\mathfrak{N}_{n} \ominus L_{\delta} \mathfrak{N}_{n}$. Set $\mathfrak{M}=\sum_{j=1}^{\infty} \oplus \mathfrak{M}_{j} \oplus \sum_{n=1}^{\infty} \oplus \mathfrak{N}_{n} . \quad$ By Lemma 5.5, it is clear that $\mathfrak{M}$ is a left-pure, left-invariant subspace of $\boldsymbol{H}^{2}$ with multiplicity function $m(t)$ and $\sum_{n \in Z} L_{\delta}^{n} P_{\mathfrak{F}} L_{\delta}^{*^{n}}=R_{\chi}\left(\sum_{n=1}^{\infty} F_{n}+\sum_{n=1}^{\infty} H_{n}\right)$ If $\int_{X} m(t) d \mu(t)=\mu(X)$, then $\sum_{n=1}^{\infty} F_{n}+\sum_{n=1}^{\infty} H_{n}=X$ and so $\sum_{n \in Z} L_{\delta}^{n} P_{\mathfrak{M}_{\mathcal{R}}} L_{\delta}^{*^{n}}=1$. Therefore $\mathfrak{M}$ is left-full.

(2) Suppose that $N$ is a $I_{n}$-factor. Let $m(t)$ be a measurable function on $X$ with valus in $(1 / n) \mathbb{Z}_{+}$such that $\int_{X} m(t) d \mu(t) \leqq \mu(X)$. Then there exist measurable subsets $\left\{E_{k}\right\}_{k=1}^{\infty}$ and $\left\{G_{k}\right\}_{k=1}^{n-1}$ of $X$ such that

$$
m(t)=\sum_{k=1}^{\infty} \chi_{E_{k}}(t)+\sum_{k=1}^{n-1} \frac{1}{n} \chi_{G_{k}}(t) \text {. }
$$

Then as in (1), there exists a left-pure, left-invariant subspace $\mathfrak{M}_{1}$ of $\mathbb{H}^{2}$ with multiplicity function $\sum_{k=1}^{\infty} \chi_{E_{k}}(t)$ such that $\sum_{k \in Z} L_{\delta}^{k} P_{\mathfrak{F}_{1}} L_{\delta}^{k}=R \sum_{k=1}^{\infty} \chi_{F_{k}}$ where $\mathfrak{\mho}_{1}=$ 
$\mathfrak{M}_{1} \ominus L_{\delta} \mathfrak{M R}_{1}$. Further, since $N$ is a $\mathbb{I}_{n}$-factor, there exists a family of mutually orthogonal equivalent projections $\left\{p_{j}\right\}_{j=1}^{n}$ in $N$ such that $\sum_{j=1}^{n} p_{j}=1$ and $\phi_{0}\left(p_{j}\right)=$ $1 / n$. On the other hand, since $\mu$ is non-atomic, there exists measurable subsets $\left\{G_{k, j}\right\}_{j=1}^{n-1}$ of $G_{k}$ such that $G_{k}=G_{k, 1}+G_{k, 2}+\cdots+G_{k, n-1}$ and $(1 / n) \mu\left(G_{k}\right)=\mu\left(G_{k, j}\right)$, $1 \leqq j \leqq n-1$. Then for every $k, 1 \leqq k \leqq n-1$, there exists a family of mutually disjoint measurable subsets $\left\{H_{k}\right\}_{k=1}^{n-1}$ of $X$ such that $\mu\left(H_{k}\right)=(1 / n) \mu\left(G_{k}\right)$ and $H_{k} \subset X-\sum_{k=1}^{\infty} F_{k}$. By Lemma 5.4, there exists a left-pure, left-invariant subspace $\mathfrak{N}_{k, j}$ of $\mathfrak{M}\left(H_{k}, p_{j}\right)$ such that the multiplicity function of $\mathfrak{\Re}_{k, j}$ is $\chi_{G_{k, j}}(t)(1 / n)$ and $\sum_{k \in Z} L_{\delta}^{k} P_{\mathscr{\mho}_{k, j}} L_{\delta}^{*^{k}}=R_{\chi_{\boldsymbol{H}_{k}} p_{j}}$, where $\mathfrak{\mho}_{k, j}=\mathfrak{N}_{k, j} \ominus L_{\delta} \mathfrak{N}_{k, j}$. Put $\mathfrak{M}=\mathfrak{M}_{1} \oplus \sum_{k=1}^{n-1} \sum_{j=1}^{n} \oplus \mathfrak{N}_{k, j}$ Then, by Lemma 5.5, $\mathfrak{M}$ is a left-pure, left-invariant subspace of $\mathbb{H}^{2}$ with

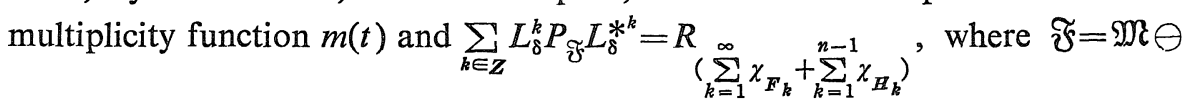
$L_{\delta} \mathfrak{M}$. This completes the proof.

As in [13, Theorem 3.1], we have the following theorem.

Theorem 5.7. Let $\mathfrak{M}$ be a left-pure, left-invariant subspace of $\mathbb{L}^{2}$ with multiplicity function $m(t)$. Then $\int_{X} m(t) d \mu(t) \leqq \mu(X)$. Furthermore, $\int_{X} m(t) d \mu(t)=$ $\mu(X)$ if and only if $\mathfrak{M}$ is left-full.

\section{§6. Case: $\mathbb{L}^{\circ}(\mathbb{X}, 8)$ is Atomic}

Throughout this section, we suppose that $L^{\infty}(X, \mu)$ is atomic. Since $\tau$ is ergodic on $X$, let $X$ be a finite set with elements $t_{0}, t_{1}, \cdots, t_{K-1}$ and let $\tau$ be the permutation of $X$ defined by $\tau\left(t_{i}\right)=t_{i+1}, i \neq K-1$, and $\tau\left(t_{K-1}\right)=t_{0}$. Further, we suppose that $\mu\left(\left\{t_{i}\right\}\right)=1,0 \leqq i \leqq K-1$, and so $\mu(X)=K$.

For $t_{i} \in X$ and a projection $p$ in $N$, we define a projection $P$ in $L(M)^{\prime}$ by

$$
(P f)\left(n, t_{j}\right)= \begin{cases}\chi_{\left(t_{i}\right)}\left(t_{j}\right) r_{p} f\left(0, t_{j}\right), & n=0, \\ 0, & n \neq 0 .\end{cases}
$$

Put $\mathfrak{M}\left(\left\{t_{i}\right\}, p\right)=\sum_{n=0}^{\infty} L_{\delta}^{n} P L_{\delta}^{*^{n}} \mathbb{L}^{2}$. Then $\mathfrak{M}\left(\left\{t_{i}\right\}, p\right)$ is a left-pure, left-invariant subpace of $\mathbb{E}^{2}$ with multiplicity function $\chi_{\left\{t_{i}\right\}}(t) \phi_{0}(p)$. Then we have the following lemmas.

Lemma 6.1. For $t_{j} \in X$, there exist a left-pure, left-invariant subspace $\mathfrak{M}_{j}$ of $\mathfrak{M}\left(\left\{t_{\imath}\right\}, p\right)$ such that multiplicity function of $\mathfrak{M}_{j}$ is $\chi_{\left\{t_{j}\right\}}(t) \phi_{0}(p)$ and $\sum_{n \in Z} L_{\delta}^{n} P L_{\delta}^{*^{n}}=\sum_{n \in Z} L_{\delta}^{n} P_{\mathfrak{\Im}_{j}} L_{\delta}^{*^{n}}=R_{\chi_{\left(t_{j}\right)}}$, where $\mathfrak{\Im}_{j}=\mathfrak{M}_{j} \ominus L_{\delta} \mathfrak{M}_{j}$. 
Proof. If $i \leqq j \leqq K-1$, put $k=j-i$ and, if $0 \leqq j<i$, put $k=j+K-i$. We note that

$$
\left(L_{\delta}^{k} P L_{\delta}^{*^{k}} f\right)\left(n, t_{l}\right)= \begin{cases}\chi_{\left\{t_{j}\right\}}\left(t_{l}\right) r_{\alpha_{0}^{k}(p)} f\left(k, t_{l}\right), & n=k, \\ 0, & n \neq k .\end{cases}
$$

Put $\mathfrak{M}_{j}=\sum_{n \geq k} L_{\delta}^{n} P L_{\delta}^{*^{n}} \mathbb{L}^{2}$. Then $\mathfrak{M}_{j}$ is clearly a left-pure, left-invariant subspace of $\mathfrak{M}\left(\left\{t_{i}\right\}, p\right)$ with multiplicity function $\chi_{\left\{t_{j}\right\}}(t) \phi_{0}\left(\alpha_{0}^{k}(p)\right)=\chi_{\left\{t_{j}\right\}}(t) \phi_{0}(p)$. This completes the proof.

Lemma 6.2. Let $E, F$ be subsets of $X$ such that $\mu(E)=\mu(F)$ and let $p$ be a projection of $N$. Then there exists a left-pure, left-invariant subspace $\mathfrak{M}$ of $\mathfrak{M}(E, p)$ such that the multiplicity function of $\mathfrak{M}$ is $\chi_{F}(t) \phi_{0}(p)$ and $\sum_{k \in Z} L_{\delta}^{k} P_{\mathfrak{F}} L_{\delta}^{*^{k}}=$ $R_{\chi_{E}} p$, where $\mathfrak{F}=\mathfrak{M} \ominus L_{\delta} \mathfrak{M}$.

Proof. Let $E=\left\{t_{i_{0}}, \cdots, t_{i_{j}}\right\}$ and $F=\left\{t_{k_{0}}, \cdots, t_{k_{j}}\right\}$. We note that $\mathfrak{M}(E, p)=$ $\sum_{l=0}^{j} \oplus \mathfrak{M}\left(\left\{t_{i_{l}}\right\}, p\right)$. Then by Lemma 6.1, there exists a left-pure, left-invariant subspace $\mathfrak{M}_{l}$ of $\mathfrak{M}\left(\left\{t_{i}\right\}, p\right)$ such that the multiplicity function of $\mathfrak{M R}_{l}$ is $\chi_{\left\{t_{k_{0}}\right\}}(t) \phi_{0}(p)$ and $\sum_{n \in Z} L_{\delta}^{n} P_{\mathfrak{M}_{l}} L_{\delta}^{*^{n}}=R_{\chi_{\left\{t_{l}\right\}} p^{*}}$. Since $\left\{\mathfrak{M}_{l}\right\}_{l=0}^{j}$ are mutually orthogonal, put $\mathfrak{M}=\sum_{l=0}^{j} \oplus \mathfrak{M}_{l}$. Then, by Lemma $5.5, \mathfrak{M}$ is a left-pure, left-invariant subspace with multiplicity function $\chi_{F}(t) \phi_{0}(p)$. This completes the proof.

Theorem 6.3. (1) Let $N$ be a III-factor. Let $m(t)$ be a function on $X$ having values in the non-negative real numbers. If $m(t)$ has the property that $\sum_{t \in X} m(t) \leqq K$, then there exists a left-pure, left-invariant subspace $\mathfrak{M}$ of $\mathbb{H}^{2}$ with multiplicity function $m(t)$. Moreover, if $\sum_{t \in X} m(t)=K$, then $\mathfrak{M}$ is left-full.

(2) Let $N$ be a $I_{n}$-factor and let $m(t)$ be a function on $X$ having values in $(1 / n) \mathbb{Z}_{+}$. If $m(t)$ has the property that $\sum_{t \in X} m(t) \leqq K$, then there exists a left-pure, left-invariant subspace $\mathfrak{M}$ of $\mathbb{H}^{2}$ with multiplicity function $m(t)$. Moreover, if $\sum_{t \in X} m(t)=K$, then $\mathfrak{M}$ is left-full.

Proof. (1) Since $N$ is a $\Pi_{1}$-factor and $\sum_{t \in X} m(t) \leqq K$, there exists a family of orthogonal projections $\left\{p_{j}\right\}_{j=0}^{K-1}$ in $N$ such that $\phi_{0}\left(p_{j}\right)=(1 / K) m\left(t_{j}\right)$ and $p_{0}+$ $p_{1}+\cdots+p_{K-1} \leqq 1$. For every $j, 0 \leqq j \leqq K-1$, we consider a family of left-pure, left-invariant subspaces $\left\{\mathfrak{M}\left(\left\{t_{i}\right\}, p_{j}\right)\right\}_{i=0}^{K-1}$. Then, by Lemma 6.1, there exists a left-pure, left-invariant subspace $\mathfrak{M R}_{j, i}$ of $\mathfrak{M}\left(\left\{t_{i}\right\}, p_{j}\right)$ such that the multiplicity function of $\mathfrak{M}_{j, i}$ is $\chi_{\left\{t_{i}\right\}}(t) \phi_{0}\left(p_{j}\right)$ and $\sum_{n \in Z} L_{\delta}^{n} P_{\mathfrak{F}_{j, i}} L_{\delta}^{*^{n}}=R_{\chi_{\left\{t_{i}\right\}_{j}}}$, where $\mathfrak{F}_{j, i}=$ $\mathfrak{M}_{j, i} \ominus \mathcal{L}_{\delta} \mathfrak{M}_{j, i}$. Since $\left\{\mathfrak{M}_{j, i}\right\}_{i=0}^{K-1}$ are mutually orthogonal, put $\mathfrak{M}_{j}=\sum_{i=0}^{K-1} \oplus \mathfrak{M}_{j, i}$. 
By Lemma 5.5, $\mathfrak{M}_{j}$ is a left-pure, left-invariant subspace of $\sum_{i=0}^{K-1} \oplus \mathfrak{M}\left(\left\{\hat{t}_{i}\right\}, p_{j}\right)$ $\left(=\operatorname{NM}\left(X, p_{j}\right)\right)$ with multiplicity function $\mathbb{K} \chi_{\left\{t_{j}\right\}} \phi_{0}\left(p_{j}\right)=\chi_{\left\{t_{j}\right\}} m\left(t_{j}\right)$ and $\sum_{n \in Z} \mathbb{L}_{\delta}^{n} P_{\widetilde{\mho}_{j}} L_{\delta}^{*^{n}}$ $=R_{p_{j}}$, where $\mathfrak{F}_{j}=\mathfrak{M R}_{j} \ominus \mathbb{L}_{\delta} \mathfrak{M R}_{j}$. Since $\left\{\mathfrak{M}\left(X, p_{j}\right)\right\}_{j=0}^{K-1}$ are mutually orthogonal, $\left\{\mathfrak{M R}_{j}\right\}$ is so. Thus, put $\mathfrak{M} \mathcal{L}=\sum_{j=0}^{K-1} \oplus \mathfrak{M}_{j}$ 。 By Lemma $5.5, \mathfrak{M}$ is a left-pure, leftinvariant subspace of $\sum_{j=0}^{K-1} \oplus \mathfrak{M}\left(X, p_{j}\right)\left(=\mathfrak{M}\left(X, \sum_{j=0}^{K-1} p_{j}\right) \subset \mathbb{H}^{2}\right)$ with multiplicity function $m(t)$ and $\sum_{n \in Z} L_{\delta}^{n} P_{\mathscr{F}} L_{\delta}^{*^{n}}=R_{\sum_{j=0}^{K-1} p_{j}}$, where $\mathfrak{F}=\mathbb{M} \ominus \mathbb{L}_{\delta} \mathfrak{M}$.

(2) Let $N$ be a $\mathbb{I}_{n}$-factor. Then there exists a family of mutually orthogonal equivalent projections $\left\{e_{l}\right\}_{l=0}^{n-1}$ in $N$ such that $\sum_{l=0}^{n-1} e_{l}=1$. Since $m(t)$ has values in $(1 / n) \mathbb{Z}_{+}$, there exists subsets $\left\{\mathbb{E}_{l}\right\}_{l=1}^{K}$ and $\left\{G_{l}\right\}_{l=1}^{n-1}$ such that

$$
m(t)=\sum_{l=1}^{K} \chi_{E_{l}}(t)+\sum_{l=1}^{n-1} \frac{1}{n} \chi_{G_{l}}(t)
$$

Put $\sum_{l=1}^{K} \mu\left(E_{l}\right)=\mathbb{L}$. As in the proof of Theorem 5.5(2), by Lemma 6.2, there exists a left-pure, left-invariant subspace $\mathfrak{M R}_{1}$ of $\mathfrak{M}\left(\left\{t_{0}, \cdots, t_{L-1}\right\}, 1\right)$ such that the multiplicity function of $\mathfrak{M E}_{1}$ is $\sum_{l=1}^{\Gamma} \chi_{E_{l}}(t)$ and $\sum_{l \in \mathbb{Z}} \mathbb{L}_{\delta}^{l} \mathbb{P}_{\mathfrak{F}_{1}} \mathbb{L}_{\delta}^{* l}=\mathbb{R}_{\chi_{\left\{t_{0}, \cdots, t_{L-1}\right\}}}$ ? where $\mathfrak{F}_{1}=\mathfrak{M R}_{1} \ominus \mathbb{L}_{\delta} \mathfrak{M E}_{1}$.

On the other hand, $\sum_{l=1}^{n-1} \frac{1}{n} \mu\left(G_{l}\right) \leqq \mathbb{K}-\mathbb{L} . \quad$ Put $\mu\left(G_{l}\right)=d_{l}$ and $G_{l}=\left\{t_{l, 1}, \cdots\right.$, $\left.t_{l, d_{l}}\right\}, 1 \leqq l \leqq n-1$. Further, set $\mathfrak{M C}_{n j+l}=\operatorname{MMC}\left(\left\{t_{L+j}\right\}, e_{l}\right), 0 \leqq j \leqq K-\mathbb{L}-1,0 \leqq l$ $\leqq n-1$. For $k$ such that $0<k \leqq d_{l}, 1 \leqq l \leqq n-1$, there exists a left-pure leftinvariant subspace $\mathfrak{R}_{l, k}$ of $\mathfrak{M R}_{d_{1}+d_{2}+\cdots+d_{l-1}+k}$ with multiplicity function $\chi_{\left\{t_{l, k}\right\}}(1 / n)$. Put $\mathfrak{N}_{l}=\sum_{k=1}^{d_{l}} \oplus \mathfrak{N}_{l, k}$. Then $\mathfrak{N}_{l}$ is a left-pure, left-invariant subspace with multiplicity function $\chi_{G_{l}}(t)(1 / n)$. Therefore, set $\mathfrak{M}=\mathfrak{M}_{1} \oplus \sum_{l=1}^{n-1} \oplus \mathfrak{N} \mathfrak{R}_{l} . \quad$ By Lemma 5.5 , there exists a left-pure, left-invariant subspace of $\mathbb{H H}^{2}$ with multiplicity function $m(t)$ such that $\sum_{l \in Z} \mathbb{L}_{\delta}^{l} P_{\widetilde{\gamma}} \mathbb{L}_{\delta}^{*^{l}}=R_{\chi_{\left\{t_{0}, \cdots, t_{L-1}\right\}}}+\sum\left\{\chi_{\left\{t_{L+j}\right\}} e_{l}: 0 \leqq n j+l \leqq d_{1}+\cdots+d_{n-1}\right\}$, where $\mathfrak{F}=\mathfrak{M} \ominus \mathbb{L}_{\delta} \mathfrak{M}$.

If $\sum_{t \in X} m(t)=K$, then it is clear that $M \mathfrak{R}$ is left-full. This completes the proof.

As in Theorem 5.7, we have the following.

Theorem 6.4. Let Me be a left-pure, left-invariant subspace of $\mathbb{L}^{2}$ with multiplicity function $m(t)$. Then $\sum_{t \in X} m(t) \leqq K$. Furthermore, $\sum_{t \in X} m(i)=K$ if and only if $\mathfrak{M e}$ is left-full. 


\section{§7. Canomical Models of Hnvariant Sublospaces}

Keep the notations and the assumptions in $\S \S 4,5$ and 6. In [6], the first author introduced the notion of canonical models defined to be a family of left-full, left-pure, left-invariant subspaces $\left\{\mathfrak{M l}_{i}\right\}_{i \in I}$ with:

(a) for no two distinct indices $i$ and $j, P_{\mathfrak{M}_{i}}$ is unitarily equivalent to $P_{\mathfrak{M}_{j}}$ by a unitary operator in $\Re$; and

(b) for every left-pure, left-invariant subspace $\mathfrak{M}$, there is an $i \in \mathbb{I}$ and a partial isometry $V$ in $\Re$ such that $V P_{\mathfrak{M}_{i}} V^{*}=P_{\mathfrak{M}}$, so that $\mathfrak{M}=V \mathfrak{M}_{i}$.

In this section we consider the canonical models of invariant subspaces in the settings of $\S \S 4,5$ and 6 . Thus we consider the following four cases:

(1) $L^{\infty}(X, \mu)$ is nonatomic and $N$ is a $\mathbb{I}_{1}$-factor;

(2) $L^{\infty}(X, \mu)$ is nonatomic and $N$ is a $\mathbb{I}_{n}$-factor, $n<\infty$;

(3) $\quad L^{\infty}(X, \mu)$ is atomic and $N$ is a $\mathbb{I I}_{1}$-factor; and

(4) $\quad L^{\infty}(X, \mu)$ is atomic and $N$ is a $\mathbb{I}_{n}$-factor, $n<\infty$.

Case (1). Put $S=\left\{m: X \rightarrow \mathbb{R}_{+}\right.$: measurable function on $X$ such that $\left.\int_{X} m(t) d \mu(t)=\mu(X)\right\}$. By Theorem 5.6, for every $m \in S$, there exists a left-pure, left-full, left-invariant subspace $\mathfrak{M}(m)$ of $\mathbb{H}^{2}$ with multiplicity function $m(t)$.

Theorem 7.1. The family $\{\mathfrak{M R}(m)\}_{m \in S}$ of left-pure, left-full, left-invariant subspaces of $\mathbb{H}^{2}$ is a complete set of canonical models in $\mathbb{L}^{2}$.

Proof. (a) is clear by Theorem 3.1.

(b) Let $\mathfrak{M}$ be a left-pure, left-invariant subspace of $\mathbb{L}^{2}$ with multiplicity function $m(t)$. By Theorem 5.7, $\int_{X} m(t) d \mu(t) \leqq \mu(X)$. Then there exists a measurable function $m_{1} \in S$ such that $m(t) \leqq m_{1}(t)$ a.e. By Theorems 3.1 and 5.6, we are done. This completes the proof.

Remark 7.2. Let $\mathfrak{M}$ be a proper two-sided invariant subspace of $\mathbb{L}^{2}$. By [11, Theorem 3.2], $\mathfrak{M}$ is left-pure and left-full. Put $\mathfrak{F}=\mathfrak{M} \ominus L_{\delta} \mathfrak{M}$. Then $P_{\mathfrak{F}} \in$ $L(M)^{\prime} \cap R(M)^{\prime}$. By [11, Lemma 3.1], there is a family $\left\{E_{n}\right\}_{n \in Z}$ of measurable sets such that $\left(P_{\mho_{\gamma}}, f\right)(n, t)=\chi_{E_{n}}(t) f(n, t)$. Therefore, $m(t)=\sum_{n \in Z} \chi_{E_{n}}(t) \in \mathbb{Z}_{+}$. Since there exists an $m \in S$ such that $m(t) \notin \mathbb{Z}_{+}$a.e., we cannot find a complete set of canonical models among the two-sided invariant subspaces.

Case (2). Put $S=\left\{m: X \rightarrow(1 / n) \mathbb{Z}_{+}\right.$: measurable function on $X$ such that $\left.\int_{X} m(t) d \mu(t)=\mu(X)\right\}$. By Theorem 5.6, for every $m \in S$, there exists a left-pure, left-full, left-invariant $\mathfrak{M}(m)$ of $\mathbb{H}^{2}$ with multiplicity function $m(t)$. 
Theorem 7.3. $\quad\{M(m)\}_{m \in S}$ is a complete set of canonical models in $\mathbb{L}^{2}$.

Remark 7.4. If $n=1$, then Solel in [13] proved that a complete set of canonical models does not consist of two-sided invariant subspaces of $\mathbb{L}^{2}$. As in $\operatorname{Remark} 7.3$, in case $n \neq 1$, we cannot find a complete set of canonical models among the two-sided invariant subspaces.

Case (3). Put $S=\left\{m: X \rightarrow \mathbb{R}_{+}: \sum_{t \in X} m(t)=\mathbb{K}\right\}$. Then, by Theorem 6.3, for $m \in S$, there exists a left-pure, left-full, left-invariant subspace $\mathfrak{M}(m)$ of $\mathbb{H}^{2}$ with multiplicity function $m(t)$.

Theorem 7.5. $\{\mathfrak{M}(m)\}_{m \in S}$ is a complete set of canonical models in $\mathbb{I}^{2}$.

Case (4). Put $S=\left\{m: X \rightarrow(1 / n) \mathbb{Z}_{+}: \sum_{t \in X} m(t)=K\right\}$. By Theorem 6.3, for $m \in S$, there exists a left-pure, left-full, left-invariant subspace $\mathfrak{M}(m)$ of $\mathbb{H}^{2}$ with multiplicity function $m(t)$.

Theorem 7.6. $\{\mathfrak{M}(m)\}_{m \in S}$ is a complete set of canonical models in $\mathbb{H}^{2}$.

Remark 7.7. If $n=1$, that is, $N=\mathbb{C}$, then the first author in [6] proved that a complete set of canonical models consists of two-sided invariant subspaces. We now suppose that $\alpha_{0}^{K}=1$. By the proof of [11, Lemma 4.2], we have $L(M)^{\prime} \cap$ $R(M)^{\prime} \subset l^{\infty}(X) \otimes 1_{N} \otimes B\left(l^{2}(\mathbb{Z})\right)=\sum_{t \in X} \oplus\left(1_{N} \otimes B\left(l^{2}(\mathbb{Z})\right)\right.$. Let $\mathbb{M}$ be a two-sided invariant subspace of $\mathbb{L}^{2}$, then $\mathbb{P}_{\mathfrak{F}} \in \mathbb{L}(M)^{\prime} \cap \mathbb{R}(M)^{\prime}$, where $\mathfrak{F}=\mathbb{M} \ominus \mathbb{L} \delta \mathfrak{M}$. Thus the multiplicity function $m(t)$ of $M \mathbb{R}$ has values in $\mathbb{Z}_{+}$. If $n \neq 1$, then we cannot find out a complete set of canonical models among two-sided invariant subspaces.

Additions. After this paper was finished independently, the authors received a paper from B. Solel entitled by "The invariant subspace structure of nonselfadjoint crossed products" which is published in Trans. AMS, 279 (1983), 825-840. His work is more general than ours. However, canonical models of invariant subspaces from the point of view of a numerical valued function allows us for a more understandable method of constructing the actual subspaces if less general. After that, the authors also received a paper from S. Kawamura and N. Tomimori entitled by "Some families of shift operators and invariant subspaces" which is published in Bull. Yamagata Univ., $\mathbb{1} 1$ (1984), 17-31. Their paper is a generalization of [6] to their setting and so overlaps $\$ 5$ in our paper.

\section{Referengees}

[1] Dixmier, J., Von Neumann algebras (English Edition), North Holland, AmsterdamNew York-Oxford, 1981.

[2] Kawamura, S., Invatiant subspaces of shift operators of arbitrary multiplicity, $J$ Math. Soc. Japan, 34 (1982), 339-354. 
[3] _ Invariant subspaces for shift operators of multiplicity one, Tohoku Math. J., 34 (1982), 15-21.

[4] - A decomposition of reducing subspace for shift operators, Tohoku Math. $J ., 35$ (1983), 425-440.

[5] McAsey, M., Invariant subspaces of nonselfadjoint crossed products, Pacific J. Math., 96 (1981), 4.57-473.

[6] - Canonical models for invariant subspaces, Pacific J. Math., 91 (1980), 377-395.

[7] McAsey, M., Muhly, P.S. and Saito, K.-S., Nonselfadjoint crossed products (Invariant subspaces and maximality), Trans. Amer. Math. Soc., 248 (1979), 381-409.

[8] — Nonselfadjoint crossed products II, J. Math. Soc. Japan, 33 (1981), 485495.

[9] - Nonselfadjoint crossed products III (Infinite algebras), J. Operator Theory. 12 (1984), 3-22.

[10] Saito, K.-S., Invariant subspaces for finite maximal subdiagonal algebras, Pacific J. Math., 93 (1981), 431-434.

[11] — Automorphisms and nonselfadjoint crossed products, Pacific J. Math., 102 (1982), 179-187.

[12] Segal, I.E., A non-commutative extension of abstract integration, Ann. of Math., 57 (1953), 401-457.

[13] Solel, B., Multiplicity functions of invariant subspaces for nonselfadjoint crossed products, to appear in Pacific J. Math.

[14] Takesaki, M., Duality for crossed products and the structure of von Neumann algebras of type III, Acta Math., $\mathbb{1 3 1}$ (1973), 249-308.

[15] - Theory of operator algebras I, Springer-Verlag, Berlin-Heiderberg-New York, 1979. 